\title{
Characterization of solitary pulmonary nodules: Use of washout characteristics at contrast-enhanced computed tomography
}

\author{
XIAO-DAN YE ${ }^{1 *}, J_{A N-D I N G ~ Y E^{1 *}, Z^{2} \text { ZHENG YUAN }}^{2,3}$, SHENG DONG $^{4}$ and XIANG-SHENG XIAO ${ }^{4}$ \\ ${ }^{1}$ Department of Radiology, Shanghai Chest Hospital Affiliated to Shanghai Jiaotong University, Shanghai 200030; \\ ${ }^{2}$ Department of Radiology, Fudan University Shanghai Cancer Center, Shanghai 200032; ${ }^{3}$ Department of Radiology, \\ Nanjing Jinling Hospital, Clinical School of Medical College, Nanjing University, Nanjing, Jiangsu 210002; \\ ${ }^{4}$ Department of Radiology, Affliated Changzheng Hospital, The Second Military Medical University, \\ Shanghai 200003, P.R. China
}

Received August 24, 2011; Accepted November 28, 2011

DOI: $10.3892 / \mathrm{ol} .2011 .520$

\begin{abstract}
The purpose of this study was to determine whether computed tomographic scans and attenuation measurements on contrast material-enhanced and non-enhanced computed tomographic scans could be used to characterize solitary pulmonary nodules and, in particular, to characterize these lesions using washout characteristics on contrast-enhanced computed tomography. A total of 63 patients (38 males, 25 females; age range, 21-80 years; mean age, $58 \pm 13.2$ years) with pulmonary nodules revealed on contrast-enhanced computed tomography underwent 20-min delayed enhanced scans. The mean diameter of the pulmonary nodules was $1.8 \pm 0.6 \mathrm{~cm}$ (range, 0.8-2.9). Region-of-interest measurements were obtained at non-enhanced, dynamic enhanced and delayed enhanced computed tomography and were used to calculate a relative percentage washout as follows: 1 - (Hounsfield unit measurement on delayed image/Hounsfield unit measurement on dynamic image) $\mathrm{x} 100 \%$. There was a mean relative washout of $33 \%$ on the delayed computed tomographic scans (range, 12-46) in benign solitary pulmonary nodules; and a mean relative washout of 7\% (range, -36-51) in malignant solitary pulmonary nodules (Mann-Whitney $\mathrm{U}$ test, $\mathrm{p}<0.001$ ). Results of the receiver operating curve analysis revealed that a threshold relative washout of $14.5 \%$ had $74.3 \%$ sensitivity and $92.9 \%$ specificity for identifying malignant nodules.
\end{abstract}

Correspondence to: Dr Zheng Yuan, Department of Radiology, Fudan University Shanghai Cancer Center, 270 Dong An Road, Shanghai 200032, P.R. China

E-mail: yuanzheng0404@163.com

Dr Sheng Dong, Department of Radiology, Affiliated Changzheng Hospital, The Second Military Medical University, 415 Feng Yang Road, Shanghai 200003, P.R. China

E-mail: dongsheng2828@hotmail.com

*Contributed equally

Key words: pulmonary nodules, lung cancer, computed tomography, washout
Calculation of the relative percentage washout on dynamic and delayed enhanced computed tomographic scans may lead to a highly specific test for solitary pulmonary nodule characterization and reduce the need for, and possibly obviate, follow-up imaging or biopsy.

\section{Introduction}

A solitary pulmonary nodule (SPN) is defined as an approximately round lesion less than $3 \mathrm{~cm}$ in diameter that is completely surrounded by pulmonary parenchyma without other pulmonary abnormalities (1). Recent advanced technology, such as low-dose, helical computed tomography (CT) screening and multi-detector row $\mathrm{CT}$, has increased the incidental detection rate of SPNs. When an SPN is detected, imaging techniques may be used to characterize the nodule in terms of whether it is likely to be benign or malignant (2). CT is the modality of choice in the imaging characterization of pulmonary nodules. Chest CT is considered the standard technique for assessing morphologic findings (1-8) and the intrathoracic spread of an SPN. The evaluation of tumor vascularity by using contrast material-enhanced CT has proven to be useful for differentiating between malignant and benign nodules (9-12). Various threshold attenuation values have been reported to be useful for distinguishing malignant nodules from benign ones on contrast-enhanced dynamic CT with single- or multidetector row helical machines $(9,10,12)$. In general, malignant nodules tend to enhance substantially more than benign nodules $(9,10,12)$. However, in previous studies, which were focused on the early phase of dynamic CT scanning, a certain overlap was found between malignant and benign nodules, for example, active granulomas or benign vascular tumors $(12,13)$. Therefore, although the results of these dynamic studies showed a high sensitivity for the diagnosis of malignant nodules, the specificity was too low. In addition, approximately $50 \%$ of indeterminate lung nodules, for which diagnosis was obtained at surgery, were benign, and hospitalization for the surgical removal of these nodules was expensive and involved a certain amount of morbidity and mortality $(14,15)$. Therefore, non-invasive imaging modalities for the specific diagnosis of indeterminate lung nodules are required. 
In previous studies $(16,17)$, the washout characteristics of lesions on contrast-enhanced CT were assessed. Washout refers to the reduction of attenuation values of lesions at CT during a variable period following the intravenous injection of a bolus of contrast material. These studies added new information on the imaging characterization of SPN. According to these studies, it should be possible to generate a washout percentage that reflects the difference in attenuation measurements of SPNs obtained at dynamic and delayed contrast-enhanced CT. To the best of our knowledge, no evaluation of the accuracy of pulmonary nodule washout characterization at dynamic contrast-enhanced CT has been reported.

The purpose of the present study was to assess the accuracy of the relative percentage washout characteristics with dynamic contrast-enhanced multi-detector row CT to distinguish malignant SPNs from benign ones.

\section{Materials and methods}

Patients. The patients included in this study fulfilled the following criteria: solitary nodule without satellite nodules; longest diameter $<3 \mathrm{~cm}$; approximately spherical; short- and long-axis diameters within a factor of 1.5 of each other; no therapy prior to examination; no benign (diffuse, laminated, popcorn-like or central) patterns of calcification; no fat on thin-section CT; nodules without ground glass opacity on thin-section CT; satisfactory patient respiratory registration without artifact on equatorial images; no marked reaction to contrast medium that interfered with image acquisition. Our institutional review board approved our research protocol for this CT study, and written informed consent was obtained from all patients.

A total of 63 patients ( 38 males and 25 females; age range, 21-80 years, mean 58 \pm 13.2 years) with an SPN at chest radiography underwent dynamic chest $\mathrm{CT}$.

CT examination. CT examinations were performed by using a 16-detector row (Toshiba Aquilion) scanner. Before dynamic CT was performed, we obtained targeted thin-section helical CT scans (1.0-mm collimation, $0.4 \mathrm{sec}$ gantry rotation time, $120 \mathrm{kVp}, 200 \mathrm{~mA}$ ) from the lung apices to the level of the middle pole of the kidneys for tumor staging. Image data were reconstructed with a thickness of $2.0 \mathrm{~mm}$ by using standard and lung algorithms, respectively. Initial dynamic scanning was performed with a 90 -sec scanning delay from the beginning of the bolus administration of contrast material. Delayed scanning limited to the nodule area was performed $20 \mathrm{~min}$ following initiation of the administration of contrast material by using similar scanning parameters and without moving the patient from the scanning table. Image data following enhancement were reconstructed with a thickness of $2.0 \mathrm{~mm}$ by using a standard algorithm.

For each lesion, regions of interest (ROI) were drawn around the lesion on each image of the non-enhanced, dynamic and delayed CT scans, and then the mean CT value was calculated. Each ROI measurement was performed twice by two investigators (Z.Y. and X.Y.), and the mean value was extrapolated to minimize error. Furthermore, each scanner was calibrated daily against a water phantom to ensure accurate attenuation measurements.
Table I. Diagnoses of 63 nodules.

Nodule type and diagnosis No. of nodules (\%)

\section{Malignant}

Adenocarcinoma

42 (66.7)

Squamous cell carcinoma

28

Bronchioloalveolar cell carcinoma

6

Mucoepidermoid carcinoma

Small cell carcinoma

Metastatic carcinoma

2

1

1

Benign

4

Follow-up with imaging

$21(33.3)$

Tuberculosis

14

Hamartoma

3

Wegener's granulomatosis

Fungal granulomatosis

Bronchial cysts

1

1

1

1

From the mean Hounsfield unit (HU) value in each ROI of the pulmonary nodules on the dynamic and delayed CT scans, a relative percentage washout was calculated as follows: 1 - (HU measurement on delayed scan/HU measurement on dynamic scan) $x 100 \%$. The absolute delayed attenuation measurements on delayed scans and absolute enhanced attenuation on dynamic scans were recorded to determine the amount of lesions that could be characterized by these methods. In addition, the accuracy of these methods were compared with that of the calculated relative percentage washout values.

Statistical analysis. Statistical analysis was performed with SPSS software (version 10.0, SPSS, Inc.). The values were compared between malignant and benign nodules by use of the Mann-Whitney U test. Receiver operating characteristic analysis was performed to determine a threshold for use in differentiating malignant nodules from benign nodules. Two-sided tests were used and $\mathrm{P}<0.05$ was considered to indicate a statistically significant difference.

\section{Results}

Of 63 SPNs, $42(66.7 \%)$ proved to be malignant and $21(33.3 \%)$ proved to be benign (Table I). The mean diameter of the nodules was $1.8 \mathrm{~cm}$ (range, 0.8-1.9).

Non-enhanced CT. The mean CT attenuation value in 21 benign lesions on non-enhanced CT scans was $37 \pm 7.7 \mathrm{HU}$ (range, 32-57). The mean attenuation value in 42 malignant

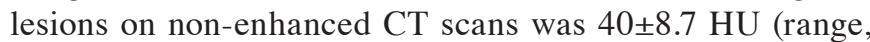
16-59). The mean attenuation value of benign nodules on nonenhanced CT scans was not significantly different from that of malignant nodules (Mann-Whitney U test, $\mathrm{p}=0.068$ ).

Early enhanced CT and wash-in of contrast material. The mean attenuation of the 21 benign lesions on early contrast-enhanced CT scans was $69 \pm 30.9$ HU (range, 34-159). Net enhancement attenuation (wash-in) was 33 $\pm 30.0 \mathrm{HU}$ (range, 0-113). 
ROC Curve

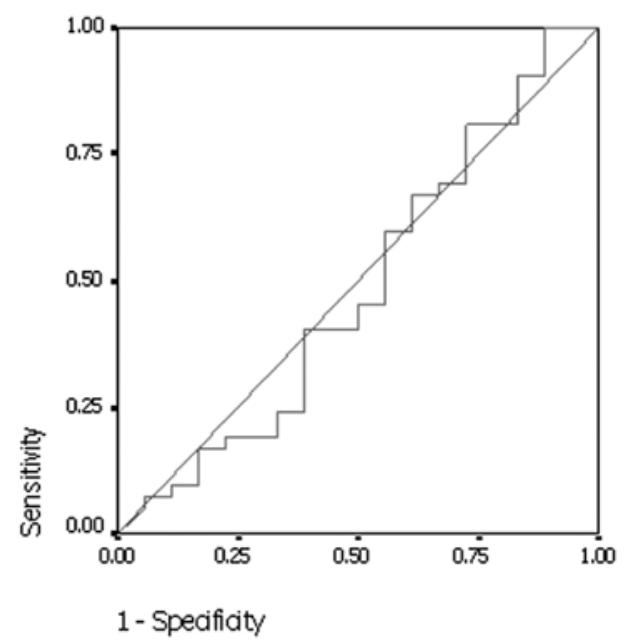

Figure 1. The graph shows the results of the receiver operator characteristic (ROC) analysis for differentiating between malignant and benign nodules with net enhancement attenuation on early dynamic contrast-enhanced CT. Area under curve is $0.484, \mathrm{p}=0.847$. CT, computed tomography.

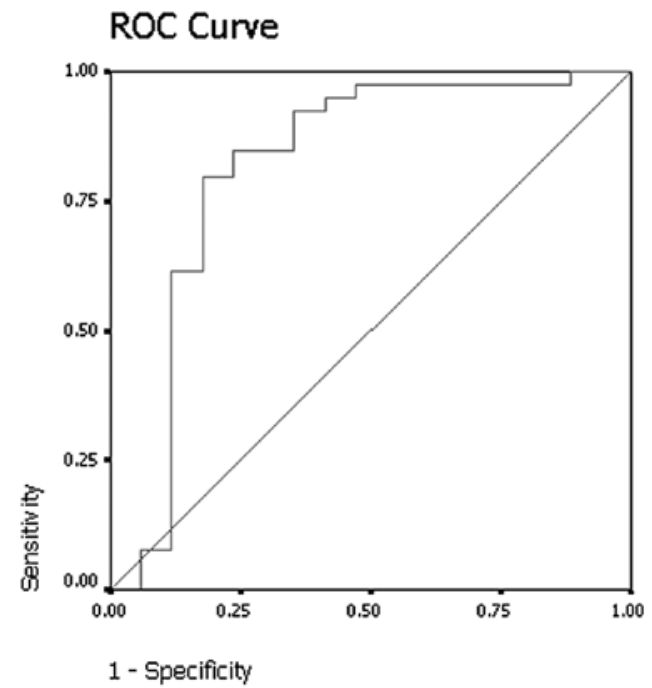

Figure 2. The graph shows the results of the receiver operator characteristic (ROC) analysis for differentiating between malignant and benign nodules with mean absolute attenuation at delayed contrast-enhanced CT. Area under curve is $0.816, \mathrm{p}<0.001$. CT, computed tomography.

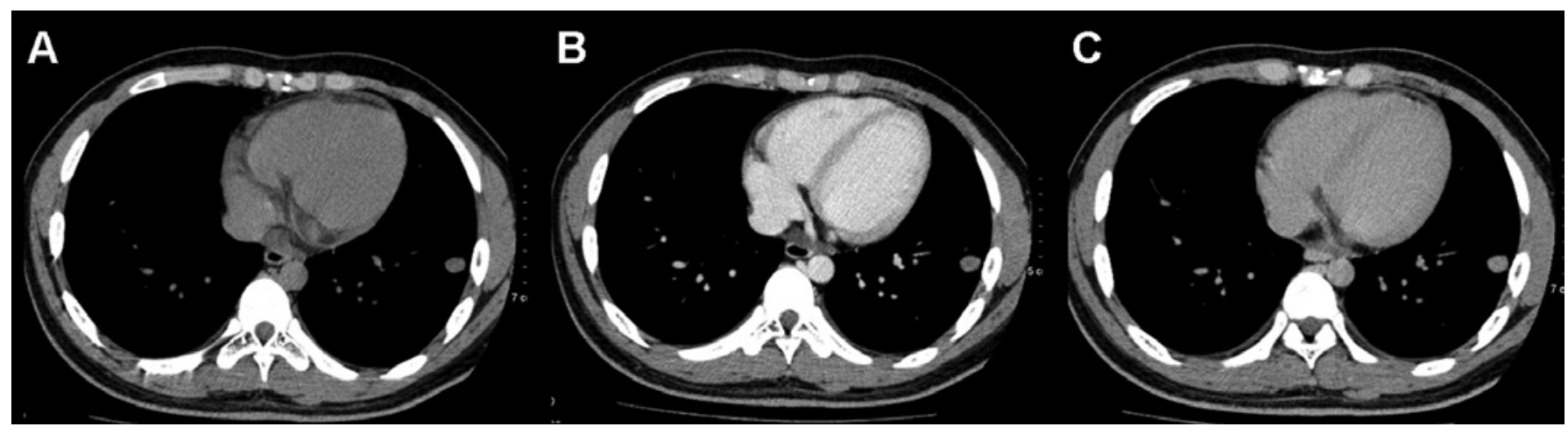

Figure 3. CT scans of Wegener's granulomatosis in a 21-year-old male. (A) Non-enhanced, (B) early enhanced and (C) delayed enhanced CT scan transverse images obtained through a nodule in the left lower lobe. The CT attenuation values on serial scans are $31 \mathrm{HU}, 56 \mathrm{HU}$ and $38 \mathrm{HU}$, respectively. Net enhancement is $25 \mathrm{HU}$; relative percentage washout, $33 \%$. CT, computed tomography.

The mean attenuation of the 42 malignant lesions on early

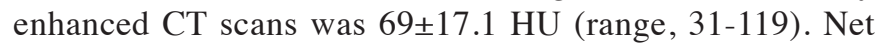
enhancement attenuation (wash-in) was 29+17.9 HU (range, 0-96). No significant differences were found between benign and malignant nodules with regard to the mean attenuation value (Mann-Whitney $U$ test, $\mathrm{p}=0.384$ ) and net enhancement attenuation (wash-in) (Mann-Whitney $U$ test, $\mathrm{p}=0.847$ ) on early contrast-enhanced CT scans.

Results of the receiver operating curve analysis (Fig. 1) showed that a threshold net enhancement value of $25 \mathrm{HU}$ had $40.5 \%$ sensitivity and $55.6 \%$ specificity for identifying malignant nodules on early contrast-enhanced CT scans.

Delayed contrast-enhanced $C T$ and relative percentage washout of contrast material. On delayed contrast-enhanced CT scans, the mean absolute attenuation value for the 21 benign nodules was $48 \pm 16.8 \mathrm{HU}$ (range, 27-93). The mean absolute attenuation value for the 42 malignant nodules on delayed contrast-enhanced CT scans was 62 \pm 11.7 HU (range, 36-92). The absolute attenuation values of malignant nodules were significantly larger than values of benign nodules on delayed contrast-enhanced CT scans (Mann-Whitney U test, $\mathrm{p}<0.001$ ). Results of receiver operating curve analysis (Fig. 2) showed that a threshold absolute attenuation value of $55 \mathrm{HU}$ had $82.1 \%$ sensitivity and $76.5 \%$ specificity for identifying malignant nodules on delayed contrast-enhanced CT scans.

On the delayed contrast-enhanced CT scans, by excluding 4 nodules that demonstrated net enhancement attenuation values of $<10 \mathrm{HU}$, the mean relative percentage washout value for benign nodules was 33\% (range, 12-46) (Fig. 3). By excluding 4 lesions that demonstrated net enhancement attenuation values of $<10 \mathrm{HU}$, the mean relative percentage washout value for malignant nodules was 7\% (range, -36-51) (Fig. 4). The attenuation values of 12 of the malignant lesions actually increased on the delayed scan. The relative percentage washout values of malignant nodules were significantly lower than the values of the benign nodules on delayed contrast-enhanced CT scans (Mann-Whitney U test, $\mathrm{p}<0.001)$. The results of receiver operating curve analysis showed that a threshold relative percentage washout of $14.5 \%$ 


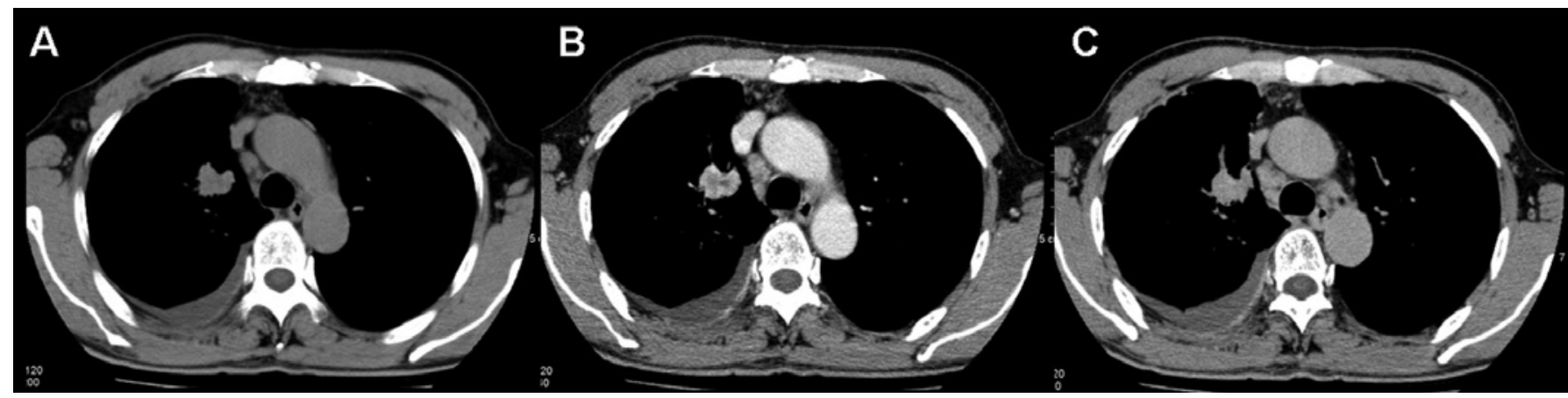

Figure 4. CT scans of bronchioloalveolar carcinoma in a 61-year-old male. (A) Non-enhanced, (B) early enhanced and (C) delayed enhanced CT scan transverse images obtained through a nodule in the right upper lobe. The CT attenuation values on serial scans are $36 \mathrm{HU}, 75 \mathrm{HU}$ and $92 \mathrm{HU}$, respectively. Net enhancement is $39 \mathrm{HU}$; relative percentage washout, $-23 \%$. CT, computed tomography.

ROC Curve

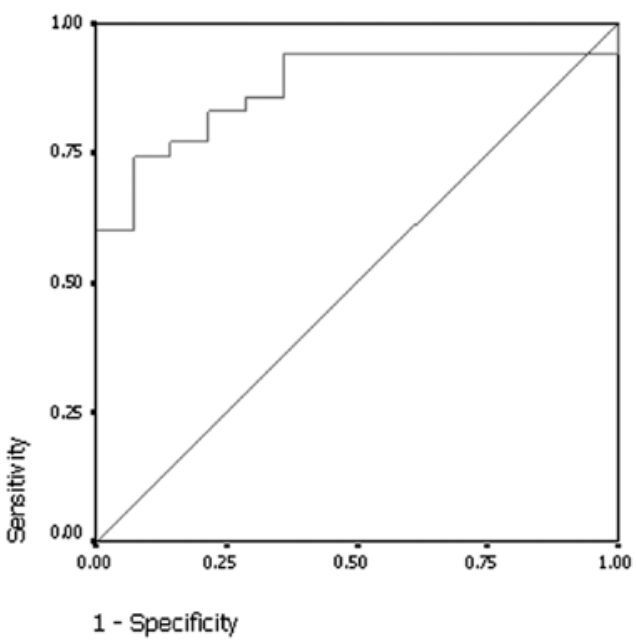

Figure 5. The graph shows the results of the receiver operator characteristic (ROC) analysis for differentiating between malignant and benign nodules with mean relative washout at delayed contrast-enhanced CT. Area under curve is $0.878, \mathrm{p}<0.001$. CT, computed tomography.

had $74.3 \%$ sensitivity and $92.9 \%$ specificity for identifying malignant nodules (Fig. 5).

\section{Discussion}

SPNs are caused by a variety of disorders including neoplasms, infections, inflammation, and vascular and congenital abnormalities. Although the majority of SPNs have benign causes, $30-40 \%$ of these nodules are malignant. Despite a number of clinical and radiologic features indicating the diagnosis, a number of SPNs remain indeterminate following conventional radiologic evaluation. A number of SPNs have similar features, and $25-39 \%$ of malignant nodules are inaccurately classified as benign following radiological assessment of size, margins, contours and internal characteristics (18). Patients with these lesions therefore require additional work-up to determine the diagnosis.

The evaluation of tumor vascularity by using contrast material-enhanced $\mathrm{CT}$ has proven to be useful for differentiating between malignant and benign nodules. In general, malignant nodules tend to enhance substantially more than benign nodules on CT $(9,10,12)$. Yamashita et al (19) reported that a maximum attenuation of 20-60 HU appears to be a good predictor of malignancy. In their study, Swensen et al (20) reported that a threshold value of $15 \mathrm{HU}$ produced a sensitivity of $98 \%$, a specificity of $58 \%$ and an accuracy of $77 \%$ for malignant nodules. Cut-off values for the differentiation between benign and malignant nodules have since been set at 15 or $20 \mathrm{HU}$.

However, all of these previous dynamic CT studies $(9,10,12)$ were focused on the early phase of dynamic CT scanning, and the results showed low specificities that range,d from 54 to $77 \%$. In our study, a threshold value of $25 \mathrm{HU}$ produced a sensitivity of $40.5 \%$ and a specificity of $55.6 \%$ for malignant nodules. Moreover, early-phase dynamic CT did not help to differentiate malignant nodules from active granulomas or benign vascular tumors.

A number of authors assessed the washout characteristics of adrenal lesions and pulmonary nodules on contrast-enhanced CT $(16,17,21)$. Findings now confirm the usefulness of attenuation measurements at non-enhanced and delayed contrast-enhanced CT for the differentiation of benign from malignant lesions $(16,17,21)$. In this study, we evaluated the accuracy of pulmonary nodule washout characterization at dynamic contrast-enhanced CT. The relative percentage washout values in the malignant nodules were significant lower than those in the benign nodules $(\mathrm{p}<0.001)$. We also found that a threshold relative washout of $14.5 \%$ had $74.3 \%$ sensitivity and $92.9 \%$ specificity for identifying malignant nodules by receiver operating curve analysis. The results showed a higher specificity using washout characterization than that for wash-in characterization in the early phase of dynamic CT scanning. The biological basis for the observed difference in washout characterization in malignant and benign pulmonary nodules can be postulated. Transduction of contrast medium through the lung involves the intravascular and interstitial spaces (22). A large interstitial space has been found in certain malignant tumors. Of note, in the washout phase from the interstitial space, a near absence or substantial reduction of lymphatic flow is noted in malignant tumors (22). The retarded flow in the intravascular and interstitial spaces is likely to contribute to the retention of contrast medium in malignant nodules. Outflow of contrast medium (washout) through the intravascular space in benign nodules, particularly in an inflammatory situation, occurs through relatively straight vessels with a normal config- 
uration. Additionally, washout of the contrast medium from the interstitial space is accelerated by active lymphatic flow (23). In the inflammatory nodules, the time-attenuation curve declines after reaching peak height, due to normal washout (13). In malignant nodules, the curve changes little after reaching peak height, due to the retarded flow in the washout phase.

There were several limitations to our study. First, pathological proof was not obtained for a number of the benign nodules. However, follow-up CT scans helped to diagnose benign nodules by showing no growth or a decrease in the size of the nodules.

Second, the previous study showed that certain nodules had persistent enhancement on 15-min delayed CT scanning (16). Therefore, we selected a 20-min delay, so as to leave enough time for the washout of contrast material from a pulmonary nodule. Although the application of washout threshold values may provide high sensitivity and specificity in differentiating between benign and malignant nodules, we found that it is difficult to obtain a delayed scan at precisely 20 min due to the pressures of the CT schedule.

Third, no pathophysiological data or proof is presented to explain the washout characteristics of benign and malignant nodules.

In conclusion, the evaluation of SPNs by analyzing washout characteristics at dynamic multi-detector row CT was proved useful for differentiating between benign and malignant nodules However, further studies are required to determine a suitable and effective delayed CT scan protocol for clinical practice.

\section{References}

1. Ost D, Fein AM and Feinsilver SH: The solitary pulmonary nodule. N Engl J Med 348: 2535-2542, 2003.

2. Shaffer K: Role of radiology for imaging and biopsy of solitary pulmonary nodule. Chest 116: 519-522, 1999.

3. Tan BB, Flaherty KR, Kazerooni EA, Iannettoni MD and American College of Chest Physicians: The solitary pulmonary nodule. Chest 123: S89-S96, 2003.

4. Winer-Muram HT: The solitary pulmonary nodule. Radiology 239: 34-49, 2006.

5. MacMahon H, Austin JH, Gamsu G, Herold CJ, Jett JR, Naidich DP, Patz EF Jr, Swensen SJ and Fleischner Society: Guidelines for management of small pulmonary nodules detected on CT scans. A statement from the Fleischner Society. Radiology 237: 395-400, 2005.

6. Christensen JA, Nathan MA, Mullan BP, Hartman TE, Swensen SJ and Lowe VJ: Characterization of the solitary pulmonary nodule: 18F-FDG PET versus nodule-enhancement CT. Am J Roentgenol 187: 1361-1367, 2006.
7. Marom EM: CT of the solitary pulmonary nodule - a commentary. Am J Roentgenol 190: 1154-1155, 2008.

8. Matsuoka S, Kurihara Y, Yagihashi K, Niimi H and Nakajima Y: Peripheral solitary pulmonary nodule: CT findings in patients with pulmonary emphysema. Radiology 235: 266-273, 2005.

9. Swensen SJ, Brown LR, Colby TV and Weaver AL: Pulmonary nodules: CT evaluation of enhancement with iodinated contrast material. Radiology 194: 393-398, 1995.

10. Swensen SJ, Brown LR, Colby TV, Weaver AL and Midthun DE: Lung nodule enhancement at CT: prospective findings. Radiology 201: 447-455, 1996

11. Yamashita K, Matsunobe S, Takahashi R, Tsuda T, Matsumoto K, MikiH, Oyanagi H and Konishi J: Small peripheral lung carcinoma evaluated with incremental dynamic CT: radiologic-pathologic correlation. Radiology 196: 401-408, 1995.

12. Yi CA, Lee KS, Kim EA, Han J, Kim H, Kwon OJ, Jeong YJ and Kim S: Solitary pulmonary nodules: dynamic enhanced multi-detector row CT study and comparison with vascular endothelial growth factor and microvessel density. Radiology 233: 191-199, 2004.

13. Zhang $\mathrm{M}$ and Kono M: Solitary pulmonary nodules: evaluation of blood flow patterns with dynamic CT. Radiology 205: 471-478, 1997.

14. Bernard A: Resection of pulmonary nodules using video-assisted thoracic surgery. The Thorax Group. Ann Thorac Surg 161: 202-204, 1996.

15. Keagy BA, Starek PJ, Murray GF, Battaglini JW, Lores ME and Wilcox BR: Major pulmonary resection for suspected but unconfirmed malignancy. Ann Thorac Surg 38: 314-316, 1984.

16. Jeong YJ, Lee KS, Jeong SY, Chung MJ, Shim SS, Kim H, Kwon OJ and Kim S: Solitary pulmonary nodule: characterization with combined wash-in and washout features at dynamic multi-detector row CT. Radiology 237: 675-83, 2005.

17. Pena CS, Boland GW, Hahn PF, Lee MJ and Mueller PR: Characterization of indeterminate (lipid-poor) adrenal masses: use of washout characteristics at contrast-enhanced CT. Radiology 217: 798-802, 2000.

18. Gurney JW, Lyddon DW and McKay JA: Determining the likelihood of malignancy in solitary pulmonary nodules with Bayesian analysis. Part II. Application. Radiology 186: 415-422, 1993.

19. Yamashita K, Matsunobe S, Tsuda T, Nemoto T, Matsumoto K, Miki H and Konishi J: Solitary pulmonary nodule: preliminary study of evaluation with incremental dynamic CT. Radiology 194: 399-405, 1995.

20. Swensen SJ, Viggiano RW, Midthun DE, Müller NL, Sherrick A, Yamashita K, Naidich DP, Patz EF, Hartman TE, Muhm JR and Weaver AL: Lung nodule enhancement at CT: multicenter study. Radiology 214: 73-80, 2000.

21. Caoili EM, Korobkin M, Francis IR, Cohan RH, Platt JF, Dunnick NR and Raghupathi KI: Adrenal masses: characterization with combined unenhanced and delayed enhanced CT. Radiology 222: 629-633, 2002.

22. Littleton JT, Durizch ML, Moeller G and Herbert DE: Pulmonary masses: contrast enhancement. Radiology 177: 861-871, 1990

23. Dewan NA, Gupta NC, Redepenning LS, Phalen JJ and Frick MP: Diagnostic efficacy of PET-FDG imaging in solitary pulmonary nodules: potential role in evaluation and management. Chest 104: 997-1002, 1993. 